A Balloon-Borne High-Resolution Spectrometer for Observations of Gamma-Ray Emission from Solar Flares

C. J. Crannell, R. Starr, A. R. Stottlemyer, and J. I. Trombka

FEBRUARY 1984

Natıonal Aeronautics and

Space Administration

Goddard Space Flight Center

Greenbelt, Maryland 20771 
A BALLOON-BORNE HIGH-RESOLUTION SPECTROMETER

FOR OBSERVATIONS OF GAMMA-RAY EMISSION

FROM SOLAR FLARES

C. J. Crannell

Laboratory for Astronomy and Solar Physics

NASA Goddard Space Flight Center

Greenbelt, MD 20771

R. Starr

Department of Physics

The Catholic University of America

Washington, DC 20064

A. R. Stottlemyer

System Sciences Division

Computer Sciences Corporation

Silver Spring, MD 20910

and

J. I. Trombka

Laboratory for Astronomy and Solar Physics

NASA Goddard Space Flight Center

Greenbelt, MD 20771

Submitted to

Nuclear Instruments and Methods in Physics Research

February 1984 


\title{
A BALLOON-BORNE HIGH-RESOLUTION SPECTROMETER \\ FOR OBSERVATIONS OF GAMMA-RAY EMISSION \\ FROM SOLAR FLARES
}

\begin{abstract}
The design, development, and balloon-flight verification of a payload for observations of gamma-ray emission from solar flares are reported nere. The payload incorporates a high-purity germanium semiconductor detector, standard NIM and CAMAC electronics modules, a thermally stabilized pressure housing, and regulated battery power supplies. The flight system is supported on the ground with interactive data-handling equipment comprised of similar electronics hardware. The modularity and flexibility of the payload, together with the resolution and stability obtained throughout a 30-hour flight, make it readily adaptable for high-sensitivity, long-duration balloon flight applications.
\end{abstract}




\section{Introduction}

Nuclear gamma-ray line emission provides the most direct evidence for the acceleration, confinement, transport, and interactions of energetic protons and ions in solar flares. The relative intensities of the lines serve as critical diagnostics not only for the spectra of the accelerated particles but also for the constituents of the ambient media in which they interact. Observations obtained with the Gamma Ray Spectrometer (GRS) onboard the Solar Maximum Mission (SMM) suggest that energetic ions may be produced in all flares. The detailed spectral observations show, moreover, that the shape of the ion energy spectrum is relatively constant from flare to flare [1]. This evidence for the near universality of ion acceleration in solar flares reinforces the hypothesis that the information carried by these emissions is fundamental to an understanding of the flare process. Much of that information, however, is contained in the precise central energy and shape of gamma-ray emission lines. Such measurements require the fine spectroscopic resolution which can be achieved with germanium semiconductor detectors. See, for example, reference [2]. Attempts to observe gamma-ray line emission from solar flares with germanium detectors have begun only recently and have not yet been successful.

Because flares with detectable gamma-ray emission occur inf requently, observing intervals of one month or longer near the maximum of a solar cycle are required to give a high probability of detecting such events. No spacecraft missions dedicated to high-energy solar physics are planned for the next cycle, but long-duration balloon flights will be possible [3]. 
The use of balloon platforms offers many attractive features including the capability of carrying large payloads ( $\sim$ ton) for long durations ( 230 days). Scientific ballooning is operated with flexible schedules and procedures, and balloon platforms provide a low-background environment. For solar objectives, balloon platforms offer the opportunity to observe flares continuously for several hours or longer. This contrasts with the case for satellites in low Earth orbit which are eclipsed for $\sim 30$ minutes in every $\sim 95$ minute orbit. The objectives of our instrumental efforts, therefore, are to develop and verify the performance of a balloon payload with sufficient resolution and stability for the desired measurements. An additional goal is that the payload be modular and flexible so that it can be expanded readily to achieve a sensitivity comparable to that of the SMM/GRS.

The first high-resolution spectrometers to be flown were lithium-drifted germanium used by Jacobson [4] for observations of the Crab Nebula and by Womack and Overbeck [5] for the first attempt at high-resolution observations of gamma-ray emission from solar flares. Presently, high-purity germanium is being employed in both satellite and balloon-borne applications $[6,7]$. These efforts have been built on the experience and data obtained with scintillation spectrometers. This includes, in particular, the use of active shielding pioneered by frost and Rothe [8] for low-energy gamma-ray astronomy. The evolution of these instrumental techniques for hard $x$ rays and low-energy gamma rays has been reviewed by Peterson [9] and for the gamma-ray domain, by Chupp [10] and by Fichtel and Trombka [11]. 
Several of the challenges encountered on near-Earth and sub-orbital platforms, have been studied extensively. These include the background enviroment [12] and the response functions of spectrometer telescopes [13]. The challenge which we are addressing is to verify the performance of a system adaptable for long-duration balloon flights. As the data presented in the following sections demonstrate, the desired resolution and stability have been achieved.

The spectrometer is described in Section 2; the onboard and ground support systems for command and data handling, in section 3; and the mechanical and thermal components, in Section 4. In Section 5, the performance of the payload during a flight of more than 30-hours duration is presented. 


\section{Spectrometer Description}

The first flight of the system was launched on November 1, 1980, from Gimli, Manitoba, Canada. This successful engineering test flight included approximately six hours with the payload at a float altitude of $36 \mathrm{~km}$. Unfortunately, the gondola and pressure housing were demolished when the payload was dropped from a helicopter during "recovery". The system was rebuilt and flown again from Greenville, South Carolina, on June 10 and 11, 1982. The following is a description of the rebuilt system, shown in Figure 1, as it was flown in June 1982.

The heart of the gamma-ray spectrometer is an actively shielded high-purity germanium (HPGe) semiconductor detector, a spectroscopy amplifier, and an 8192 channel ADC. The HPGe detector (built by Princeton Gamma-Tech) is a right circular cylinder, $58 \mathrm{~mm}$ in diameter by $45 \mathrm{~mm}$ in height, in a standard p-type coaxial configuration. A Power Designs $A E C-5000 B$ high voltage power supply was used to positively bias the detector at 3300 volts during operation. The detector preamplifier was powered with a Tennelec TC205A spectroscopy amplifier which also served as the pulse-shaping amplifier for the preamplifier output.

Cooling to a temperature of approximately $77 \mathrm{~K}$ for the HPGe and the field effect transistor (FET) in the detector preamplifier is provided by liquid nitrogen contained in a 30-liter dewar. A copper rod is used to transfer heat from the crystal and the FET to the liquid nitrogen. The capacity of the dewar allows an operating lifetime, between fills, of approximately seven days. 
The detector efficiency at $661 \mathrm{keV}$ is $25 \%$ that of a cylindrical $\mathrm{NaI}(\mathrm{T} 1)$ scintillator $7.6 \mathrm{~cm}$ in diameter by $7.6 \mathrm{~cm}$ in height. The detector resolution at $1.17 \mathrm{MeV}$ is better than $2.5 \mathrm{keV}$ full width at half maximum (FWHM).

Collimation and background reduction for the HPGe detector is provided with a CSI( $\mathrm{Na}$ ) shield and a plastic scintillator over the aperture, as shown in Figure 1. The atmospheric attenuation of gamma-rays within our range of interest limits useful observing of extraterrestrial sources to angles less than or equal to $60^{\circ}$ of the local zenith. The size and shape of the CsI $(\mathrm{Na})$ shield and the location of the HPGe detector within it, therefore, were designed to orovide a field of view of $120^{\circ} \mathrm{FWHM}$. That is, any source within $60^{\circ}$ of the detector look axis is observed witn an effective detector area greater than or equal to half the actual detector area. Sources at any greater angle are attenuated by the shield as indicated in Figure 2. The detector look axis was aligned with the zenith, and the spectrometer was operated as a transit instrument.

The $\operatorname{CsI}(\mathrm{Na})$ shield is composed of three pieces: a hexagonal annulus which intercepts radiation from sources to the side of the HPGe and two Dees, directly beneath the annulus and under the germanium housing, which intercept any radiation incident on the HPGe from below. The annulus is viewed with three photomultiplier tubes (PMTs) and the Dees with two PMTs each. All seven are type RCA 4523. The PMTs are optically coupled to tne $\mathrm{CsI}(\mathrm{Na})$ with a transparent silicone rubber (General Electric, RTV 602). The spectral resolution of the $\operatorname{CsI}(\mathrm{Na})$ scintillators in this configuration is approximately $50 \%$ over the energy range of interest. 
The efficiency for stopping $1.33 \mathrm{MeV}$ gamma-rays from a ${ }^{50} \mathrm{Co}$ source is shown in Figure 2 as a function of the incident angle of the incomming gamma ray.

The aperture plastic, which is placed above the CsI(Na) annulus, is a 6-mm thick piece of plastic scintillator. It is viewed, through light pipes, with four RCA C31016F PMTs, and operated in anticoincidence with the HPGe detector.

Each of the photomultiplier tubes used with the CsI(Na) shield and aperture plastic is individually packaged in a cylindrical housing, made of aluminum for the CsI(Na) PMTs and plastic for the aperture plastic PMTs. Included in the housing for each PMT is the dynode high voltage divider chain and preamplifier along with a mu-metal magnetic shield for the PMT. All components are held mechanically secure in the housing with a silicone rubber potting compound (Dow Corning, RTV 3111).

The entire detector subsystem which includes the HPGe detector, liquid nitrogen dewar, $\mathrm{CsI}(\mathrm{Na})$ scintillators, and aperture plastic, is held in place and supported with a frame of aluminum plates and rods as shown in Figure 1. The aluminum plates also serve as mounting platforms for the summing and differential amplifiers used with the $\operatorname{CsI}(\mathrm{Na})$ and the aperture plastic. Each section of the $\operatorname{CsI}(\mathrm{Na})$ shield and aperture plastic operates as an independent detector and has its own set of amplifiers. The summing amplifiers, differential amplifiers, and high voltage power supplies used with the $\mathrm{CsI}(\mathrm{Na})$ and plastic detectors were all flight specific modules, individually designed for this specific application. 
3. Command and Data Handling

Data acquired with the spectrometer and on the payload status are processed in three basic stages before being seen by the experimenter. The first of these is conversion from analog form to digital data packets witnin the flight electronics. The second is transmission of these packets through the telemetry communication link with the ground station. The third is conversion of the digital data packets into recognizable science and engineering form for the investigator on the ground. The telemetry link supports a data transmission rate of $20 \mathrm{kbps}$ which enables us to transmit directly a substantial amount of information for each valid event detected with the spectrometer. This includes the energy loss measured with the HPGe detector, any associated energy loss recorded individually for each of the $\operatorname{CsI}(\mathrm{Na})$ shield elements, and the time delay between any such signals from the anticoincidence shield and the primary signal from the HPGe. A schematic representation of the functional elements which comprise the flight payload and the ground support systems is shown in Figure 3. A description of the actual electronic components and how they are employed is presented in the following two subsections.

\subsection{Onboard Electronics and Data Handling}

A block diagram of the electronics is presented in Figure 4. Except for the several flight-specific units listed in the previous section, all items shown are off-the-shelf Nuclear Instrument Modules (NIM) and Computer Automated Measurement and Control (CAMAC) modules. The only change to any of these modules was made to the LeCroy 821 Quad 
Discriminator which allows us to apply external voltages to three of the four channels in the unit for the purpose of setting threshold levels. These external threshold voltages can be set independently for the HPGe, plastic aperture, and $\operatorname{CsI}(\mathrm{Na})$ shield discriminators and can be increased or decreased by command from the ground. Rates, designated $\mathrm{R}_{1}$ through $\mathrm{R}_{7}$, are monitored at various steps in the logic sequence as illustrated in the block diagram.

The electronics logic associated with the HPGe detector and aperture plastic is designed to allow only valid events to be pulse height analyzed with the LeCroy 3511, 13-bit spectroscopy ADC. A valid event in the HPGe detector $\left(R_{2}\right)$ is one that is above a lower level threshold of approximately $60 \mathrm{keV}\left(R_{1}\right)$, below an upper level threshold of approximately $7.8 \mathrm{MeV}\left(R_{3}\right)$, and in anti-coincidence with the aperture plastic $\left(R_{4}\right)$. These rates are related as follows: $R_{2}=R_{1}-R_{3}-$ $f \cdot R_{4}$, where $f$ is an efficiency factor determined by the geometry and the 400-nanosecond coincidence window. During our June 1982 flight, the value of $f$ was approximately 0.1 .

The logic associated with the three elements of the $\operatorname{CsI}(\mathrm{Na})$ shield allows us to time tag all events which occur in the shield within a 4-microsecond wide coincidence window opened by a valid event in the PGe. The time tag is established with a LeCroy 2228A/125 Octal TDC. Only those events in the $\operatorname{CsI}(\mathrm{Na})$ above a lower level threshold of approximately $200 \mathrm{keV}\left(R_{5}, R_{6}, R_{7}\right)$, and in coincidence with HPGe valid events are pulse height analyzed with a LeCroy 22498, 12-channel ADC. 
Information on the payload status which originates in analog form, (e.g. voltages, pressures, and temperatures) is monitored with two LeCroy 2232A Differential ADCs. Digital status information (e.g. ON/OFF status and fans HIGH/LOW) is monitored with a Standard Engineering Corporation PR604 Input Register.

The conversion of data into digital packets is performed under the control of a DEC LSI-11/23 microcomputer in the flight package. This microcomputer is interfaced to both the detector and the communications link through a CAMAC based system. Detector event data and housekeeping data are collected through the set of ADCs and TDCs discussed above, and stored in a packet buffer. Raw detector rates are collected from scalers (Kinetic Systems 3610 ) on a subcommutated basis, as are digital status words and clock pulses for the calculation of detector deadtimes. These are collected approximately once per 1.6 seconds. The data are stored in a frame buffer, then moved to a buffer in the CAMAC based FIFO (Kinetic Systems 3841) upon demand from the communications link frame request.

The telemetry interface converts the 16-bit parallel data in the FIFO buffer into bi-phase $L$ serial format for transmission. The RF transmission from the payload to the tracking antennas on the ground is performed with hardware supplied by the National Scientific Balloon Facility. For testing in the laboratory environment, the entire transmission link is replaced with a simple coaxial cable.

Power for the entire payload was provided by 28 Eternacell lithium batteries (model number B7901), nineteen of which were wired to supply 810 (180) ampere-hours at $+(-) 11.2$ volts, .60 (120) ampere-hours at $+(-)$ 16.8 volts and $60(90)$ ampere-hours at $+(-) 33.6$ volts. The voltages 
quoted here are the unloaded values. These power levels were selected so as to allow a minimum flight time of 45 hours and also to maintain the average current draw from any one battery below one ampere. These six voltages were in turn regulated to \pm 6 volts, \pm 12 volts and \pm 24 volts as required by standard NIM and CAMAC electronics. The regulators employ Fairchild $\mu$ A723 Monolithic Voltage Regulators and are based on the regulator circuit suggested by Fairchild [14].

The remaining nine batteries were wired to provide 270 ampere-hours at +33.6 volts and were left unregulated. These batteries supplied the power for the heater circuits and fans. The fans were operated from a separate regulator, in order to electrically isolate them from the rest of the payload.

\subsection{Ground Support System}

On tne ground, data from the balloon payload are acquired, processed, displayed, and archived through a system based on a DEC LSI-11/23 microcomputer which serves also as the development facility for flight-computer firmware. The design of the Ground Support Equipment (GSE) with modular hardware and software provides for maximum flexibility and makes optimum use of off-the-shelf components. Software used with the GSE is based on a general purpose system developed in the Laboratory for High Energy Astrophysics of the Goddard Space Flight Center to support flight instruments [15]. The GSE serves the full range of payload operations which includes subsystem development and checkout, payload integration, calibration and pre-flight performance verification, in-flight monitoring and quick-look data analysis, and post-flight data reduction. 
Serial data received with the GSE are processed first with a bit synchronizer and then with a frame synchronizer which converts the data to 16-bit parallel form. The formated data are passed to a CAMAC-based FIFO buffer from which they are accessed in packets with the microcomputer on an interrupt basis. The ways in which the digital data are processed are selected by the investigator through the use of a command translator which also executes the stored command procedures.

The GSE is equipped with two terminals, a Tektronix 4012 video terminal and a Texas Instruments $820 \mathrm{KSR}$ dot-matrix hard-copy terminal. The payload housekeeping data can be monitored with the video terminal which may be used in conjunction with a Tektronix 4631 hard copy unit. A sample status page, showing the data format employed with these devices, is presented in Figure 5. The nousekeeping data are displayed in a similar format with a Ball Brothers video monitor driven by a Kinetic Systems 3235 display driver which is continuously updated but not attached to a hard copy device. During flight, the video terminal is devoted primarily to monitoring science data and, at regular intervals, to displaying time-nistory plots of the scaler rates and selected housekeeping paraneters. A sample of such a time-history plot is presented in Figure 6. This particular display shows the variation in the temperature of the germanium preamplifier from 21:51 EDT on June 10 to 10:21 EOT June 11 which includes the transition from night to day when the heater battery power was turned off.

The principal modes of displaying the science data are as histograms, based either on spectral distributions or temporal distributions of the accumulated events. Examples of the data displays available are 
presented in Section 5 to illustrate the results obtained in the Greenville flight. Pulse-height distributions from the germanium spectrometer and from the $\operatorname{CsI}(\mathrm{Na})$ shield elements can be monitored at intervals selected by the investigator. Threshold settings on the respective discriminator levels can be adjusted should any shifts in the distributions be observed. In addition to being a monitor for the payload status and performance, the GSE can be employed simultaneously to archive all the data acquired in the telemetry stream. The data are written on magnetic tapes in a mode which is designed to alternate between two tape drives with no loss of data. The same procedures which are used to provide quick-look data can also be employed to reduce the data to a variety of formats appropriate for analysis with a larger computational facility. 
4. Mechanical and Thermal

The balloon payload is divided into two main sections. One is the pressure housing which contains the spectrometer and the NIM and CAMAC electronics. The second is the battery cage which holds the lithium batteries, regulators, radiators, main power and heater circuit relays, and the consolidated instrument package (CIP) supplied by the NSBF. The pressure housing is mounted above the battery cage (see Figure 1) and is attached to it at six equally spaced points around its circumference. The battery cage is thermally isolated from the pressure housing through the use of nylon washers at the six contact points.

Because the NIM and CAMAC electronics used in this payload are standard off-the-shelf laboratory modules and cards, they must be maintained at a pressure of at least $2 \times 10^{4} \mathrm{~Pa}$ to prevent not spots from developing in components with large power dissipation and to avoid coronal arcing around high voltage contacts. The air temperature within the pressure housing must be maintained between 0 and $50 \mathrm{C}$ to assure reliable operation.

The two NIM bins and one CAMAC crate are secured in aluminum frames which are welded to the baseplate of the pressure housing. They are located along the periphery of the baseplate surrounding the liquid nitrogen dewar. The pressure seal between the dome $(2.5-\mathrm{mm}$ thick aluminum) and the baseplate is made with a $6.4-\mathrm{mm}$ diameter rubber 0-ring in a groove near the outer edge of the $1.3-m$ diameter baseplate. Pop-out valves in the baseplate are set to open if the relative pressure inside the pressure housing exceeds approximately 1 atmosphere, $10^{5} \mathrm{~Pa}$. This 
is particularly important because the liquid nitrogen evaporates throughout the flight. Additionally, there is a pop-in valve set to open if the outside pressure exceeds that inside by $3 \times 10^{4} \mathrm{~Pa}$. In case of a complete loss of pressure at float, this pop-in valve would permit the pressure to equalize during descent and prevent the pressure housing from imploding.

The absolute pressure just before the actual launch was $1.2 \times 10^{5}$ $\mathrm{Pa}$. It dropped quickly to $10^{5} \mathrm{~Pa}$ and remained at this pressure throughout the flight.

A stable thermal environment is required inside the pressure housing for proper functioning of the electronics, and that thermal control must be achieved despite the extreme temperature conditions encountered by day and by night at altitudes above most of the Earth's atmosphere. The temperature inside the pressure housing is maintained within proper limits through the use of several complementary techniques. The outsides of the dome and the baseplate are insulated with $3.8 \mathrm{~cm}$ of ethafoam. All external parts of the payload not covered with foam are painted white to minimize the absorption of Sun light during the daytime portion of the flight. All batteries and regulators (except the fan regulator) are located in the "battery cage," outside the pressure housing. All the 'Jatteries were thermally insulated with $5 \mathrm{~cm}$ of ethafoam because the battery lifetime decreases significantly at temperatures below $-30 \mathrm{C}$. The regulators were not insulated, and had to operate in the temperature extremes encountered at nigh altitudes.

Inside the pressure housing three different methods are used to control the temperature. First, two aluminum boxes, in thermal contact 
with the baseplate, are filled just before launch with approximately 20 $\mathrm{kg}$ each of frozen "Blue Ice." The inside of the pressure housing is then flushed with dry nitrogen before it is sealed to prevent moisture condensation. The Blue Ice provides thermal inertia which helps to slow the temperature rise during the day; and because it is warmed during the day, slows the temperature decline during the night. Secondly, fans are used to circulate air through the CAMAC crate and two NIM bins. These fans may be operated at two different speeds: HIGH speed is used during the day, and LOW speed, at night. Finally, high wattage resistors are used as heaters on several components of the payload. Four such resistors (Dale, $50 \mathrm{~W}, 25 \Omega$, wired in parallel) are located on the baseplate and produced 158 watts of heater power throughout the nightime portion of the flight (approximately $91 / 2$ hours). These heaters were commanded ON and OFF from the ground. All other heater circuits are thermistatically controlled and set to go on at temperatures below $15 \mathrm{C}$. These circuits are capable of producing a maximum of 322 watts of heater power. During the flight, however, they never exceeded even half that amount.

During the actual flight, the air temperature in the pressure housing never dropped below $12 \mathrm{C}$ and never rose above $34 \mathrm{C}$ as can be seen in Figure 7. The batteries operated in the temperature range from -6 to $+30 \mathrm{C}$. 


\section{Payload Performance}

The total time at float for the June 1982 flight was 29 1/2 hours. The float altitude varied from a low of about $35 \mathrm{~km}$ to a high of about 40 $\mathrm{km}$. Throughout the entire flight, the spectrometer performed flawlessly. The two high-resolution spectra from the HPGe detector, displayed in Figure 8, demonstrate the effectiveness of the anticoincidence shield for background rejection. The upper spectrum includes all pulses detected in the HPGe detector in anti-coincidence with the aperture plastic. The lower spectrum includes the same events except those for which an event was detected in the $\mathrm{CsI}(\mathrm{Na})$ shield within a window set as part of the analysis procedure. For the case shown here, the coincidence window on the time-tagged $\operatorname{CsI}(\mathrm{Na})$ events is set at 1.6 microseconds and the pulse-height threshold is set at the lower limit of $200 \mathrm{keV}$. A sample TOC spectrum is displayed in Figure 9. The 1.6 microsecond window used during the analysis procedure corresponds to vetoing all events with a time tag. in channels 26 through 48 . Approximately $3 / 4$ of the events observed in the germanium above:500 $\mathrm{keV}$ are rejected by the CsI( $\mathrm{Na}$ ) anticoincidence requirement.

Several gamma-ray lines are, apparent in these pulse-height spectra. The most intense line, at $0.511 \mathrm{MeV}$, is produced by positron annihilation in the atmosphere. The detailed shape of this line, obtained from an integration over the entire duration of the flight, is shown in Figure 10. The measured width of $3.0 \mathrm{keV}$ illustrates the fine inherent resolution of the HPGe spectrometer and the stability of the detector system. The lines seen in Figure 8 below $200 \mathrm{keV}$ in energy are due to. 
neutron capture in the Ge itself. Other lines, at higher energies, are due to sources such as $40_{K}$ and inelastic neutron scattering from aluminum. The background lines identified in the flight data are listed with tneir probable origin in Table 1.

The gain stability of the central detector is further demonstrated in Figure 11, which is a plot of the fractional channel number, obtained from a Gaussian fit to the peak of the $0.511-M e V$ positron annihilation line, versus time. The gain of the amplifier was adjusted so that one channel in the ADC corresponds to an energy of $1 \mathrm{keV}$. From launch to cut-down, the location of the line did not vary by more than $3 / 4$ of a channel. This corresponds to a gain stability of better than $0.15 \%$. of all the engineering parameters, the temperature variation of the germanium preamplifier is correlated most strongly with the observed gain change. This correlation can be seen in a comparison of Figures 6 and 7 with Figure 11. The magnitude of the effect is approximately a $0.1 \%$ increase in gain for a decrease in temperature of $17 \mathrm{C}$. These results verify the stability and relative temperature-insensitivity of our instrumentation and reflect the efficacy of our efforts to temperature stabilize our entire payload.

The time profile of the HPGe counting rate for the energy interval 55 to $500 \mathrm{keV}$ in anticoincidence with the $\operatorname{CsI}(\mathrm{Na})$ is shown in Figure 12. The major variations in the counting rate are well correlated with cnanges in payload altitude. The gap in the data beginning at approximately 15:00 EDT and lasting for about 49 minutes was caused by lightening striking a pawer line in the tracking station. The two spikes in the profile, at 14:30 EDT (18:30 UT), June 10 and 11:00 EDT (15:00 UT) 
June 11, are two small flares. The first was an $M 1.9$, and the second, an M 3.6 as seen in soft $X$-rays with SMS-GOES [16]. Both of these flares produced detectable $x$-ray flux in the spectrometer up to approximately $130 \mathrm{keV}$.

Detailed time profiles of the flare-associated emission above $50 \mathrm{keV}$ for both of these two events are shown in Figure 13. The data are presented on time scales appropriate to the accumulation of statistically significant flare-related flux. Both events show an impulsive spike followed by gradual emission. The spectra observed for the impulsive component of the June 10 event and both components of the June 11 event are displayed in Figure 14. They have been corrected for photon absorption in the atmosphere and the aluminum pressure housing. Additional absorption corrections not included in this analysis are less important and would tend to soften the spectrum, increasing the spectral index, $\gamma$, by not more than 0.1 (see Table 2). The spectra in Figure 14 are represented by thermal spectra with temperatures corresponding to 80 $\mathrm{keV}$ for the June 10 event and $300 \mathrm{keV}$ and $125 \mathrm{keV}$ for the impulsive and gradual components, respectively, of the event on June 11 . The gradual component of the June 10 everit was too weak for meaningful analysis. Power-law fits to each of these spectra also were carried out. The values of the parameters obtained in the spectral analysis, the associated $x^{2}$, the number of free parameters, and the probability of exceeding $x^{2}$ are given in Table 2. Although these flares were not large enough to provide a detectable flux of nuclear gamma-ray line emission, they do indicate the sensitivity of the instrument. 
In summary, performance of the payload with respect to energy resolution, stability, and sensitivity was verified through a flight which included both a day to night and a night to day transition. Because of its modular, flexible design, this payload can be expanded to provide the desired sensitivity and adapted readily for a variety of applications. Efforts to develop the additional instrumental capabilities required for long-duration balloon flights are now underway. 
Acknowledgements

At the inception of this effort, R. L. Golden provided suggestions and information on the design of a themally stabilized payload. W. S. Muney and T. B. Plummer participated in all stages of this effort and acted as the Project Engineers for the first and second flights, ræspectively. R. H. Hoffman was responsible for the detailed thermal design, L. E. Orwig assisted with the design of the flight-specific electronics, and C. J. Morgan provided the detailed mechanical design. F. C. Hallberg was responsible for coordinating the technical support for the payload construction. I. C. Nash, A. D. Huffman, and K. J. Meese carried out the electrical and flight-specific electronics assembly. G. M. Miller painted the external payload structure, and P.M. Baltzell photographically documented the payload development. For both flights, W. Hopkins and R. Bussard packaged the flight and ground-support equipment so that no damage was'sustained in transit. For the second flight, the payload was constructed in the Physics Department Shop at the University of Maryland. Additional assistance was received from numerous individuals throughout the Sciences and Engineering Directorates of the Goddard Space Flight Center and other institutions including the MIT Bates Linear Accelerator, the Computer Sciences Corporation, The Catholic University of America, the National Scientific Balloon Facility, the Space Environmental Services Center of the National Oceanic and Atmospheric Administration, and the University of Maryland. Launch and flight support services were provided by personnel of the Canada Centre for Space Science of the National Research Council Canada and of the 
Space Research Team, ADGA Systems International Limited for our first flight from Gimli, Manitoba, Canada. For the second flight, launch and flight support services were provided by personnel of the National Scientific Balloon Facility, Palestine, Texas. For both flights, logistical support was provided by the NASA/Wallops Flight Center. Support in the field, for pre-launch, launch, and flight operations was provided in Greenville by H. Caulk, F. L. Lang, H. Wiehl, and Lo I Yin. The enthusiastic participation of all the individuals involved is gratefully acknowledged and greatly appreciated. 
References

[1] E. L. Chupp, Recent Advances in the Understanding of Solar Flares, Uchida, Y., Kane, S. R., Hudson, H. S., and Tanaka, K. (eds.); Komaba, Tokyo, Japan, 389 (1982), to be published in Solar Physics (1983).

[2] R. E. Lingenfelter and R. Ramaty, Physics Today 31 (1978) 40.

[3] Long Duration Balloon Flight Study Committee: A Plan for Long Duration Scientific Ballooning, White, R. S.'(Ch.), National Scientific Balloon Facility, Palestine, Texas, USA (1983).

[4] A. S. Jacobson, Doctoral Thesis University of California San Diego, La Jolla, California (1968).

[5] E. A. Womack and J. W. Overibeck, J. Geophys. Res. 75 (1970) 1811.

[6] G. H. Nakano and W. L. Imnö, Gamma Ray Spectroscopy in Astrophysics, Cline, T. L. and Ramaty, R. (eds.), NASA Technical Memorandum 79619 (1978) 529.

[7] W. Paciesas, R. Baker, D. Boclet, S. Brown, T. Cline, H. Costlow, P. Durouchoux, C. Ehrmann, N. Gehrels, J. M. Hameury, R. Haymes, 8. Teegarden, and J. Tuelier, NASA Technical Memorandum 85011 (1983), to be published in Nucl. Instr. and Methods (1983).

[8] K. J. Frost and E. D. Rothe, IRE Trans. Nucl. Sci. NS-9 (1962) 381.

[9] L. E. Peterson, Ann. Rev. Astron. and Astrophys. 13 (1975) 423.

[10] E. L. Chupp, Gamma Ray Astronomy, D. Reidel, Dordrecht, The Netherlands (1976).

[11] C. E. Fichtel and J. I. T̈rombka, Gamma Ray Astrophysics, New Insight into the Universe, NASA SP-453 (1981). 
[12] C. S. Dyer, J. I. Trombka, S. M. Seltzer, and L. G. Evans, Nucl. Instr. and Methods 173 (1980) 585.

[13] S. M. Seltzer, Nucl. Instr. and Methods 127 (1975) 293.

[14] Fairchild Camera and Instrument Corporation, Linear Division Products (1982) p. 2-94.

[15] C. H. Ehmann and A. E. Engel, IEEE Trans. Nucl. Sci NS-Z6 (November 1979).

[16] Solar-Geophysical Data 461 Part II (January 1983). 
TABLE 1. BACKGROUND LINES OBSERVED DURING JUNE 1982 BALLOON FLIGHT

$\begin{array}{cc}\begin{array}{c}\text { Peak Channel } \\ \text { Number }\end{array} & \text { Energy (keV) } \\ 54 & 53.53(6) \\ 67 & 66.70(1) \\ 140 & 139.69(5) \\ 198 & 198.31(14) \\ 239 & -- \\ 439 & 438.63(2) \\ 443 & 442.91(7) \\ 472 & 472.32(15) \\ 497 . & -- \\ 511 & 511.0034(14) \\ 582 & --- \\ 595 & 595.88(4) \\ 667 & 667.699(25) \\ 844 & 843.76(3) \\ 1015 & 1014.46(4) \\ 1371 & 1368.596(29) \\ 1463 & 1460.726(35) \\ 1782 & 1779.0(2) \\ 1811 & 1808.68(6)\end{array}$

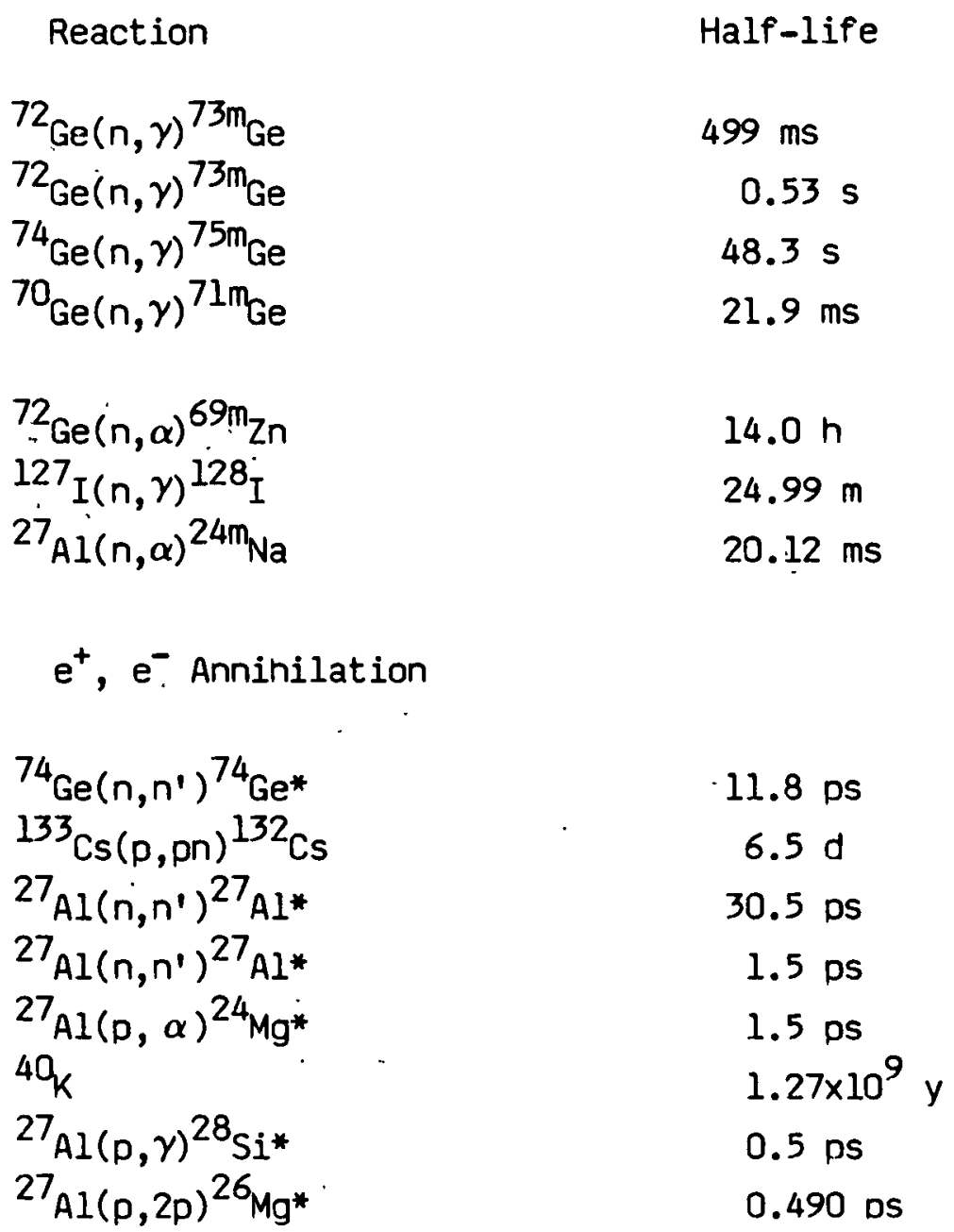


TABLE 2. RESULTS OF SPECTRAL ANALYSIS

$$
\begin{gathered}
\text { Thermal } \\
I(E)=\frac{C}{E^{1.4} T^{0.1}} e^{-E / T} \\
\text { photon } \mathrm{cm}^{-2} \mathrm{~s}^{-1} \mathrm{keV}^{-1}
\end{gathered}
$$

Event/Interval

C

$x^{2}$

$x^{2} \quad v$

$P_{x}\left(x_{v, v}^{2}\right)$

K
Power Law

$$
\begin{gathered}
I(E)=K E^{-\gamma} \\
\text { photon } \mathrm{cm}^{-2} \mathrm{~s}^{-1} \mathrm{KeV}^{-1}
\end{gathered}
$$

1982 June 10

$18: 30: 16-18: 30: 48$

$\begin{array}{rrrrr}50.7 & 80 \begin{array}{l}+36 \\ -30\end{array} \quad 9.48 & 9 & 0.40\end{array}$

$434.1 \quad 2.20 \pm 0.40 \quad 11.3$

0.25

1982 June 11

$14: 58: 34-14: 59: 23$

$20.6 \quad 300 \begin{array}{r}+700 \\ -180\end{array}$

12.911

0.30

$11.0 \quad 1.41 \pm 0.36 \quad 16.2 \quad 11$

0.18

$14: 59: 38-15: 02: 54$

$\begin{array}{llll}15.9 & 125 & +70 \\ -43 & 14.0 & 17\end{array}$

0.75

$60.91 .94 \pm 0.30$

17.9

17

0.40 
Figure Captions

la. Side view of pressure housing and battery cage. Cutaway view shows placement of CAMAC crate, liquid nitrogen dewar, and various elements of the spectrometer inside the pressure housing.

b. Top view of the electronics and spectrometer layout inside the pressure housing. Cutaway view shows HPGe inside $\mathrm{CsI}(\mathrm{Na})$ shield and below aperture plastic.

c. Top view of battery cage. Layout of ten small battery boxes (only eight visible) and large battery box is shown. The CIP sits on top of the large battery box.

2. Angular calibration of solar gamma-ray spectrometer obtained prior to Greenville flight. The detector is oriented vertically, and $90^{\circ}$ corresponds to the zenith. Uncertainties in the measured efficiencies are approximately \pm 0.05 .

3. Schematic representation of the flight payload and ground support system. This layout of the functional elements is organized to illustrate the flow within and between the flight and ground components.

4. Block diagram of the electronics directly associated with the actively shielded spectrometer.

5. Sample status page. This display, which is available both as a video display and as a hard copy, enables the investigators to monitor critical parameters associated with the payload environment and perfomance. References to LIXI describe the status of a piggyback experiment. 
6. Temperature of the germanium preamplifier as a function of time. Time history plots such as this are available through the ground support system. With the information in this readily interpretable format, the investigators are able to evaluate the trends as well as the instantaneous status of the payload throughout flight. Times listed are seconds after midnight, UT June 11.

7. Plot of selected temperatures versus time for the entire $331 / 2$ hours from launch to cut-down. The four temperatures shown are that of the air inside the pressure housing, the CAMAC crate, one NIM bin, and the HPGe preamplifier. The data are from samples taken at fifteen minute intervals. The gap near 15:00 EDT on June 10 is about 49 minutes in duration and was caused by a power failure at the tracking station.

8. High-resolution pulse height spectra from the HPGe detector accumulated for the entire $291 / 2$ hours at float altitude during Greenville balloon flight. Both spectra are for pulses from the HPGe detector in anticoincidence with pulses from the aperture plastic. For the lower spectrum, pulses from the CsI(Na) shield were also in anticoincidence. Note the factor of ten scale change on the vertical axis.

9. Histogram of events binned according to the time delay between the HPGe valid event trigger and a signal from the hexagonal annulus of the $\operatorname{CsI}(\mathrm{Na})$ shield. The time interval shown here is that of the second flare observed during the flight. Each channel is approximately 70 nanoseconds in width. 
10. The measured pulse height spectrum in the vicinity of the $0.511-$ MeV line accummulated for the full $291 / 2$ hours at float altitude. The FWHM of the line is $3 \mathrm{keV}$.

11. The fractional channel number corresponding to the peak of the $0.511-\mathrm{MeV}$ line as a function of time for the total duration of the balloon flight. The peak was determined from a gaussian fit to the spectrum after the underlying continuum emission was subtracted.

12. Count rate of the HPGe detector (between 55 and $500 \mathrm{keV}$ ) as a function of time for the entire $291 / 2$ hours at float altitude. The counts are summed over 90 second intervals. The two spikes in the data at 14:30 EDT, June 10 and 11:00 EDT, June 11 are M class flares. The gap in the data at approximately 15:00 EDT on June 10 is about 49 minutes in duration and was caused by a power failure at the tracking station.

13. Time-intensity profiles of the counting rate above $50 \mathrm{keV}$ observed from solar flares on June 10 and 11, 1982. The quiet-Sun background has been subtracted, but no corrections having to do with the instrument response function have been applied to the observed counting rate.

14. Spectra of the observed photon flux from the flares of 1982 June 10 and 11. The total observed flux (circles) and the background (squares) which was subtracted also are shown for comparison, plotted against the right hand ordinate in units of counts $\mathrm{s}^{-1} \mathrm{keV}^{-1}$. The dashed lines represent thermal spectra of the form $I(E)=\frac{C}{E^{1.4} T^{0.1}} e^{-E / T}$ photons $\mathrm{cm}^{-2} \mathrm{~s}^{-1} \mathrm{keV}^{-1}$ which give the best acceptable fit to the observations. The solid lines represent power law spectra of the form $I(E)=K E^{-\gamma}$ photons $\mathrm{cm}^{-2} \mathrm{~s}^{-1} \mathrm{keV}^{-1}$ which give the best acceptable fit to the data. 
Figure 1
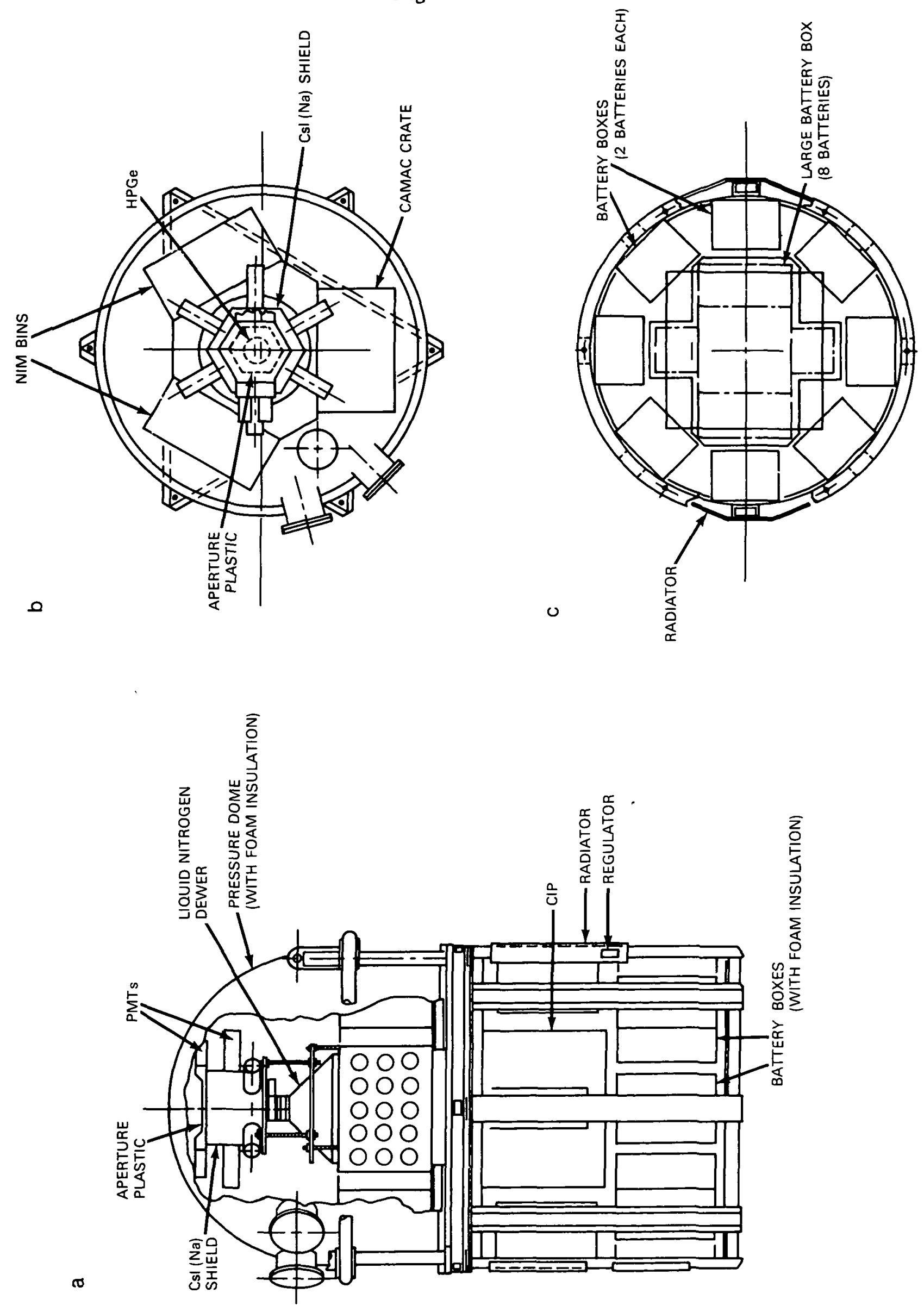
Figure 2

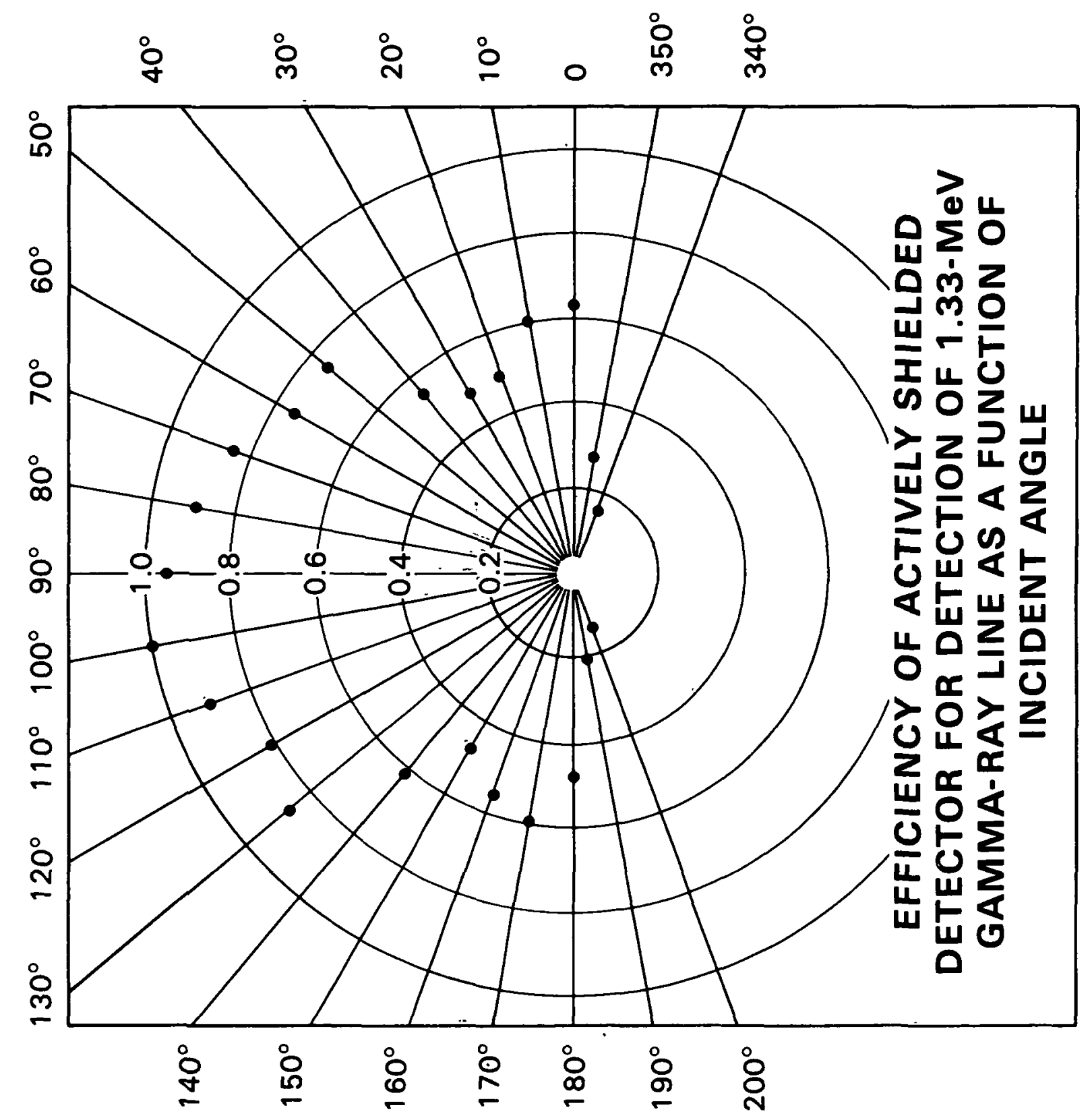


Figure 3

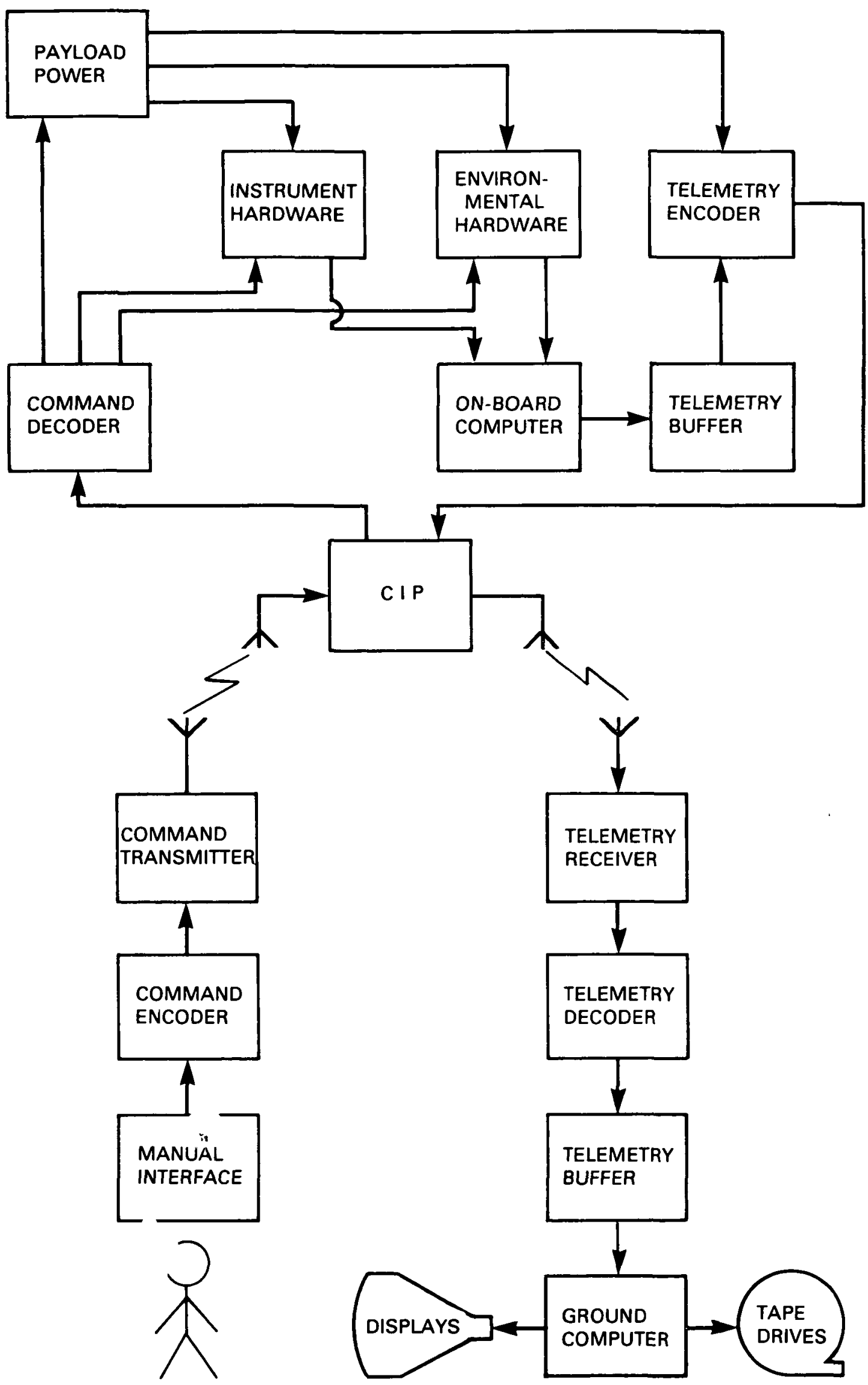


Figure 4

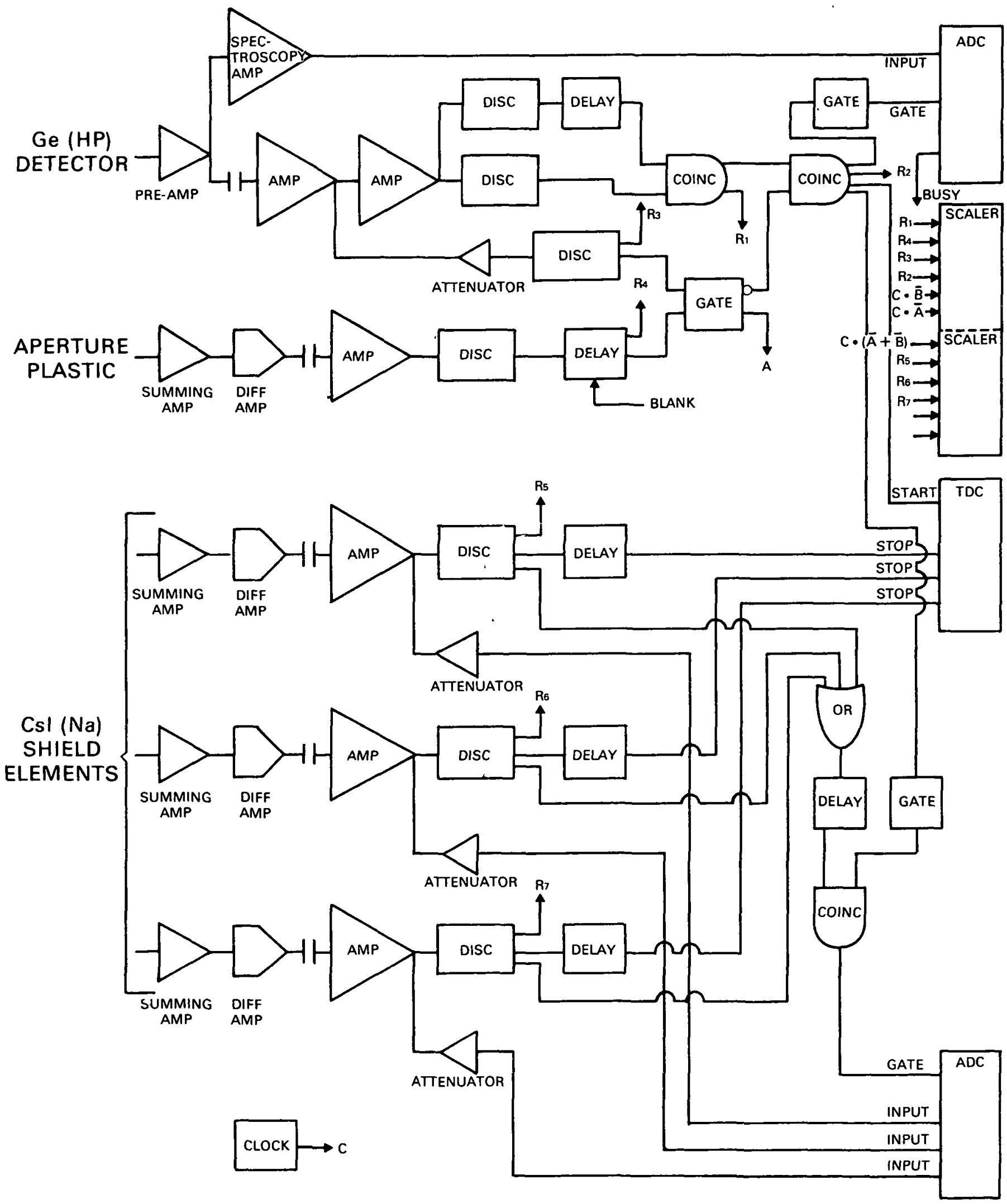


Figure 5

o

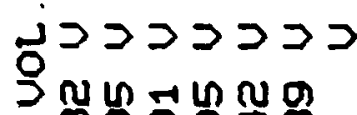

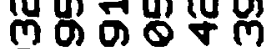

$\stackrel{\theta}{\nabla}$

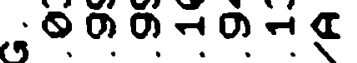
जि कित लिष

व

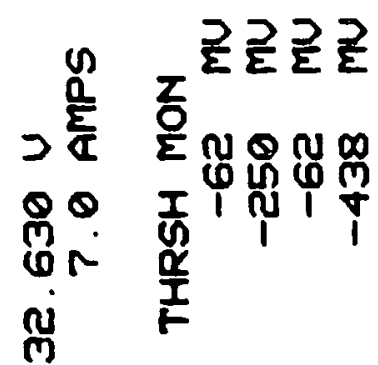

ta

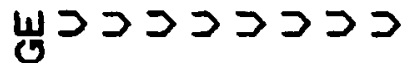

$5>25$

芒

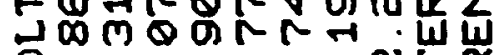

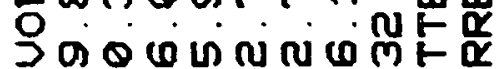

क

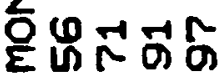

केत

至नल

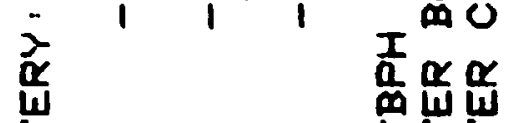

虽

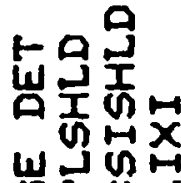

E

I

$m+1+1+1$ u $\frac{1}{1}$ 饪 w

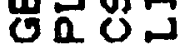

00000000000

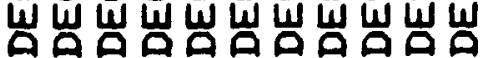

$\infty \pi+\infty \mathbb{N}+r+\boldsymbol{\theta}$

-

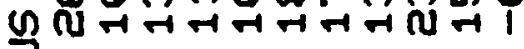

㟧

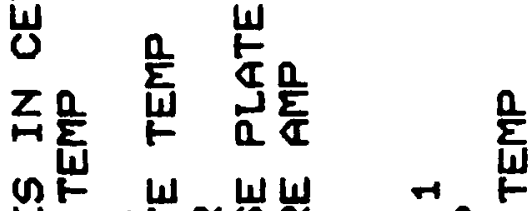
行

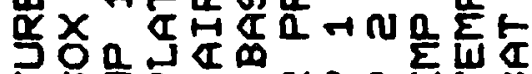

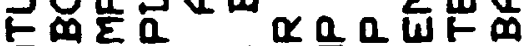

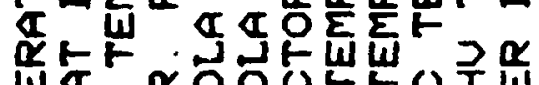

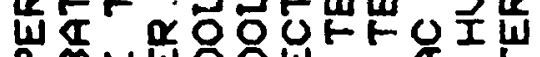

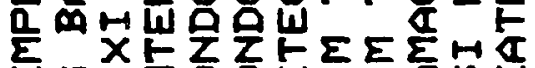

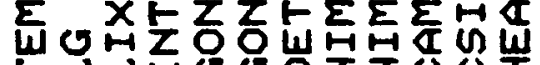

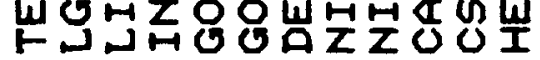

cos or 000 \%

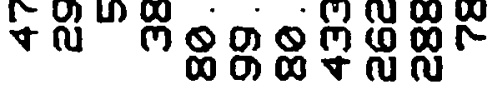

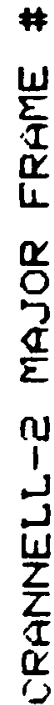

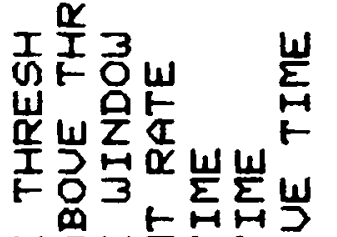

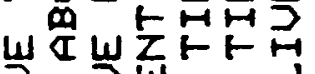

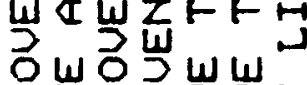

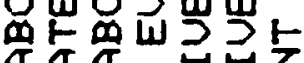

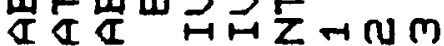

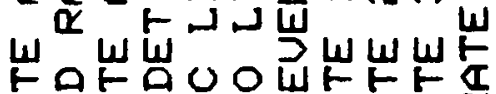

$\pi] \frac{\pi}{\alpha} 0$ a $\frac{\pi}{\alpha} \alpha$

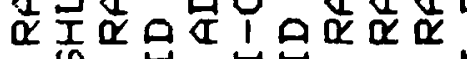

เ

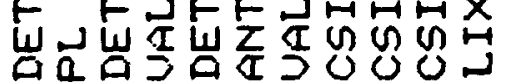

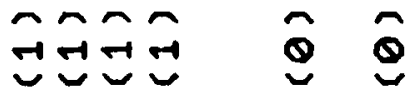

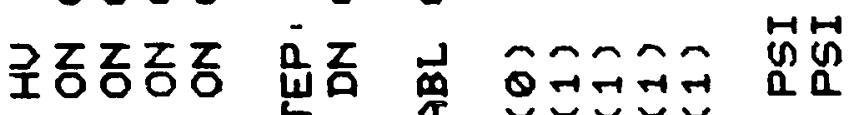

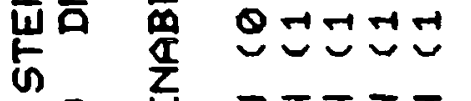

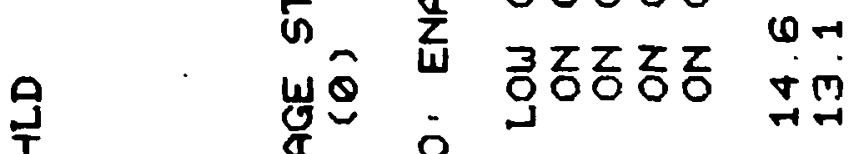

壳ñAr

$\frac{\alpha}{I}$

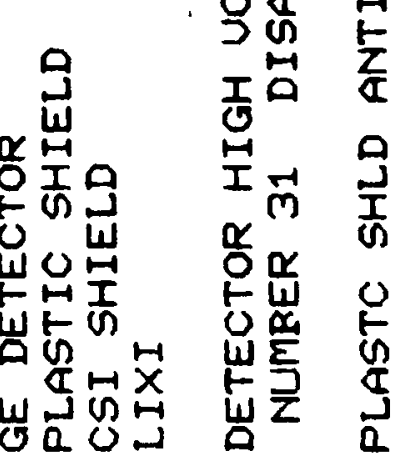

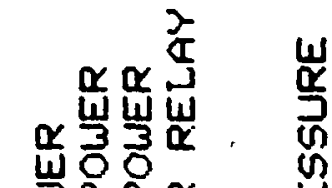

$\alpha \overline{0}$ a $\frac{\alpha}{\omega}$

눈 $\alpha \frac{w}{3}$

3 틍

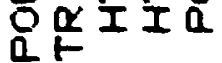

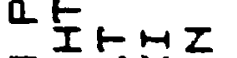

Z舟沗

$\tau \frac{w}{\alpha}$

$\frac{1}{0}$

an

突出

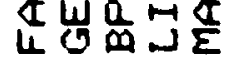


Figure 6

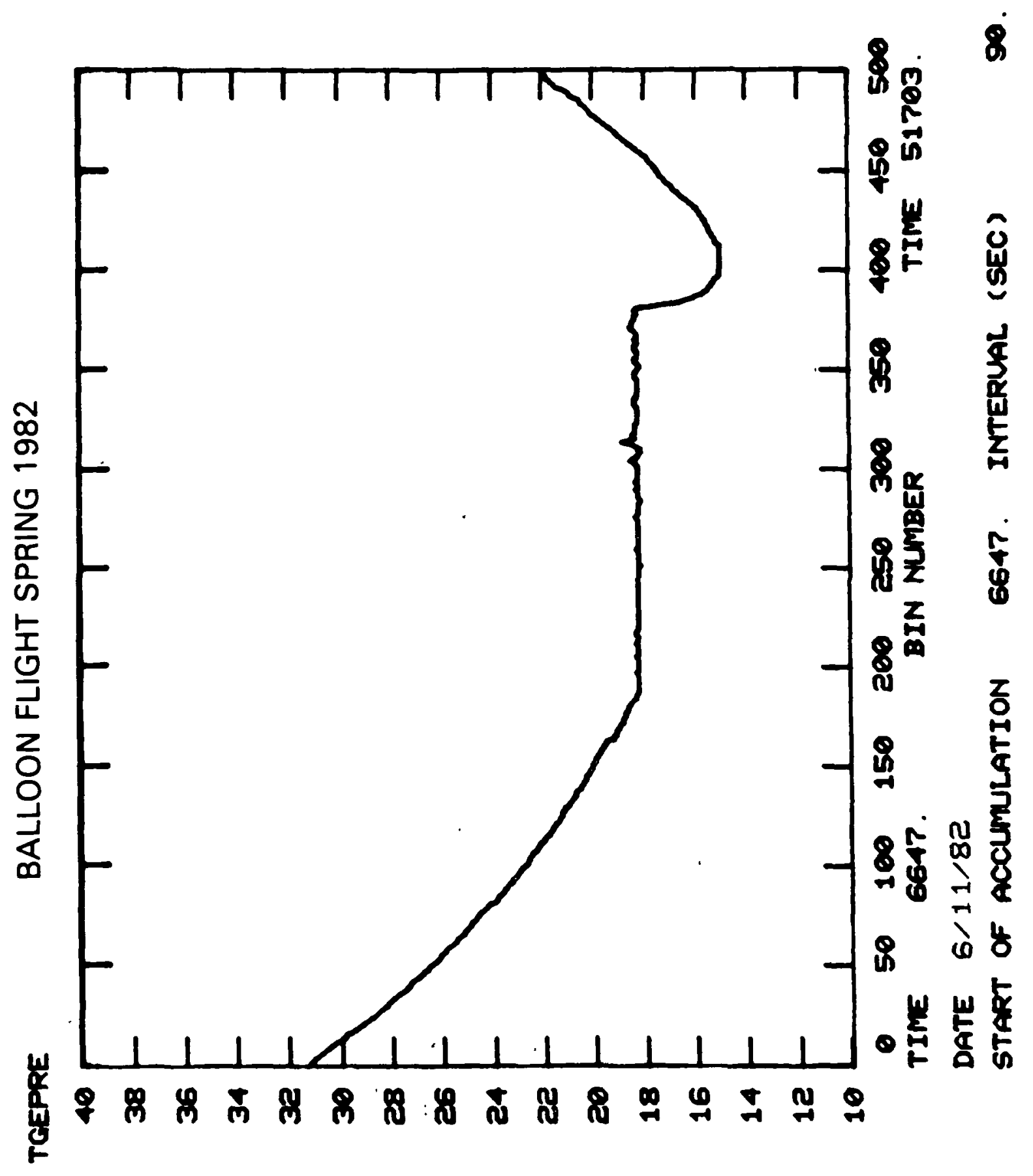

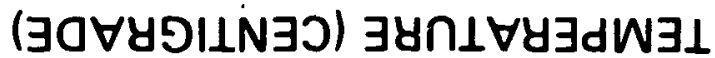


Figure 7

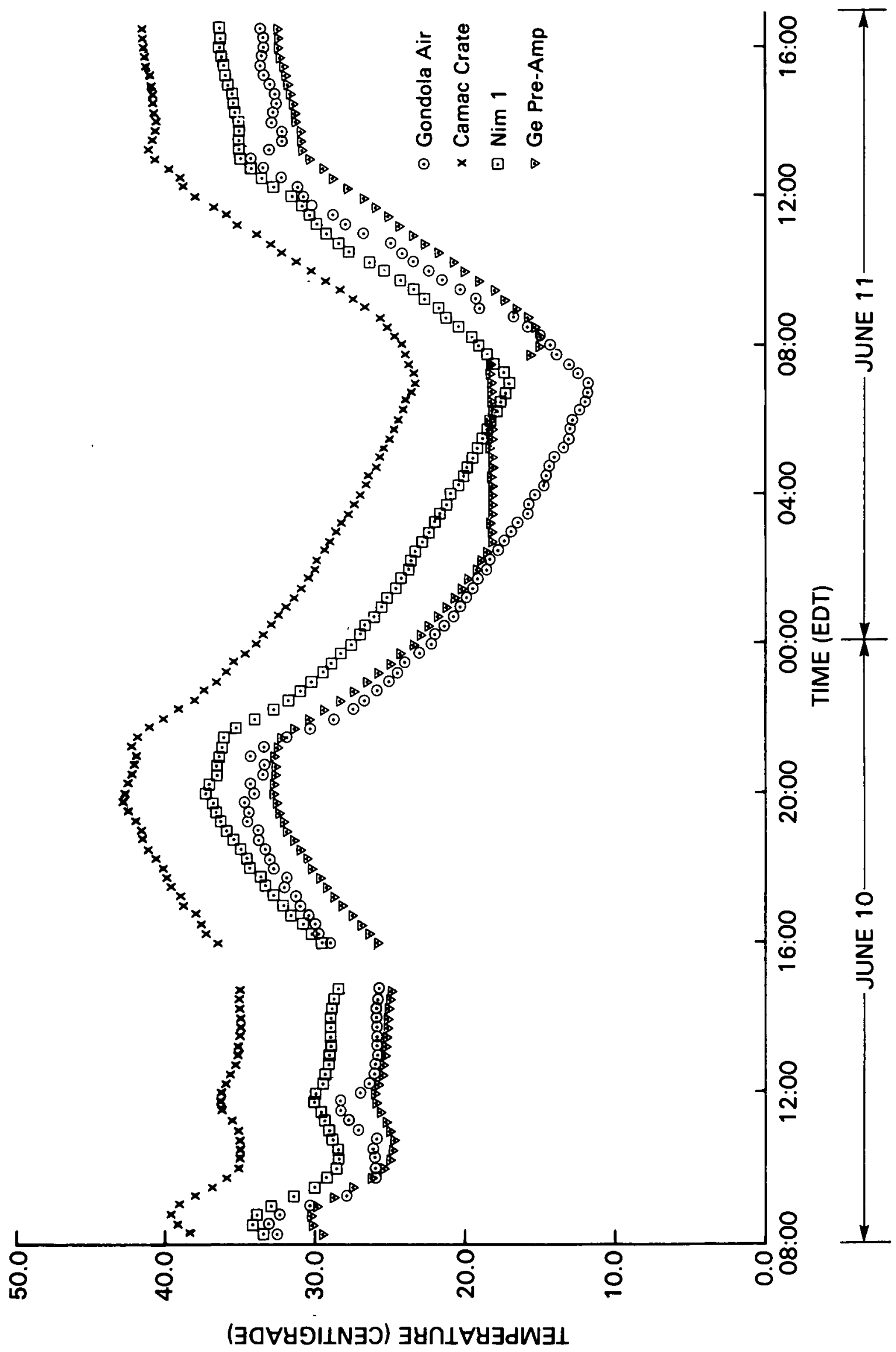




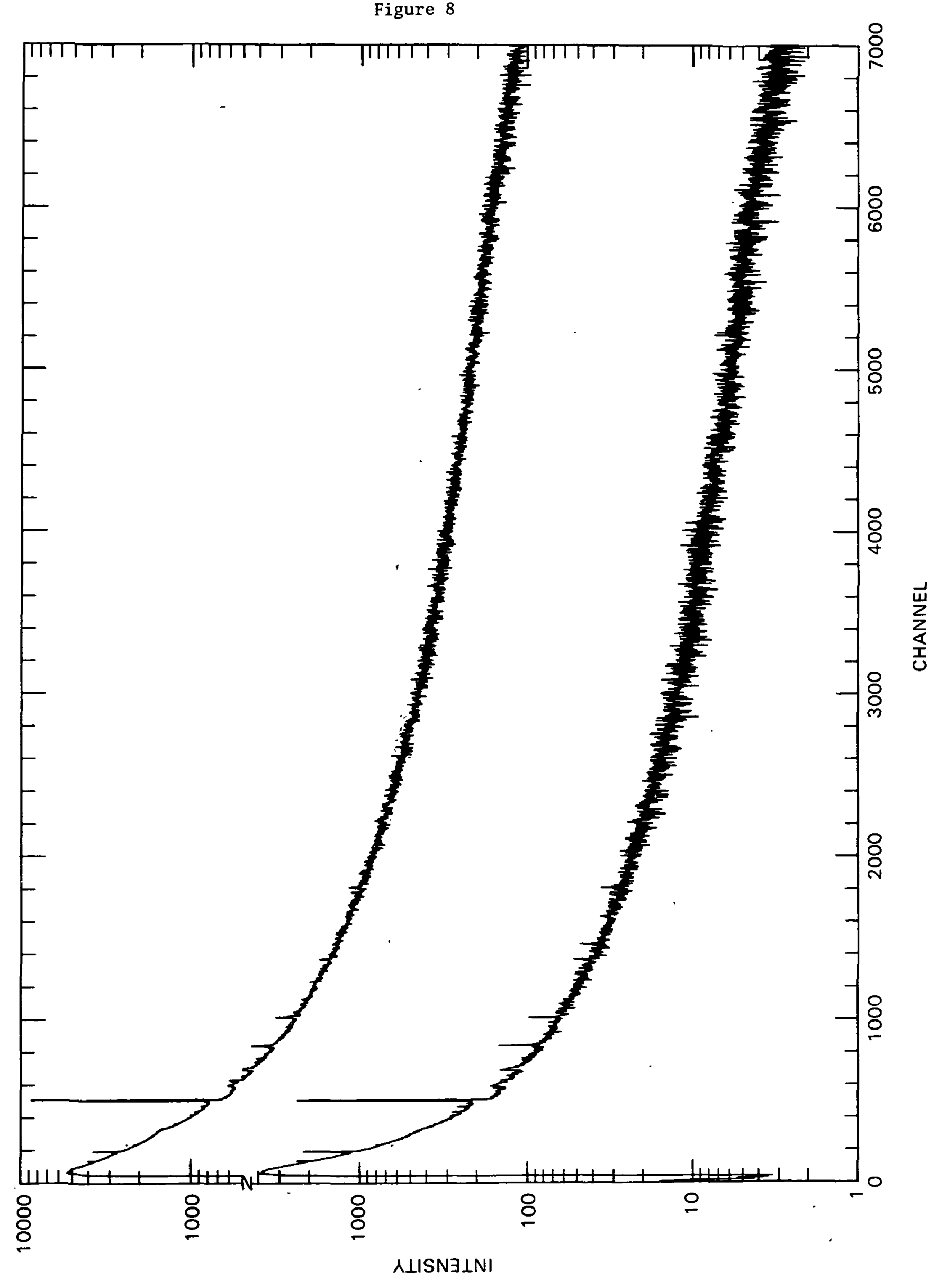


Figure 9

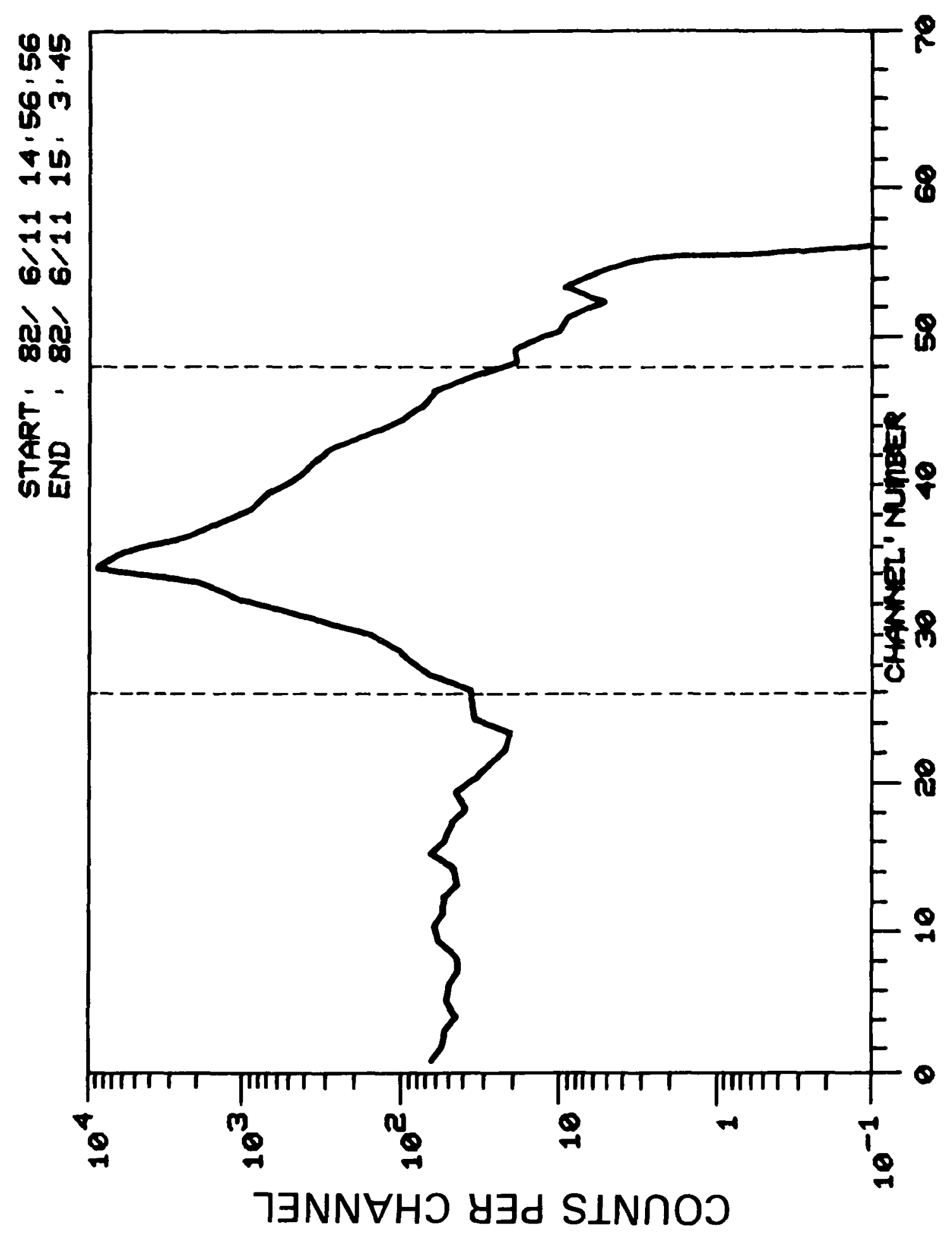


Figure 10

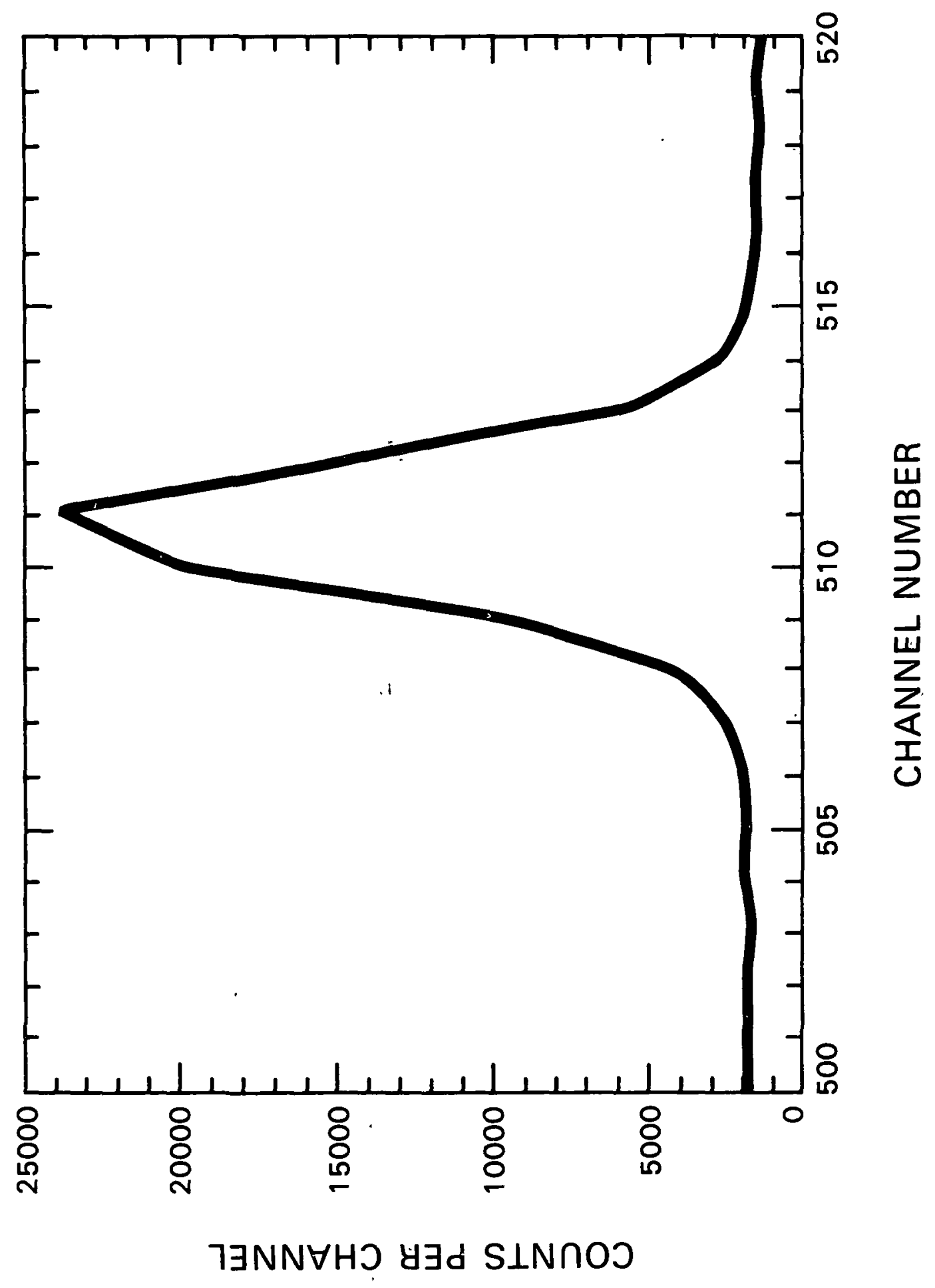


Figure 11

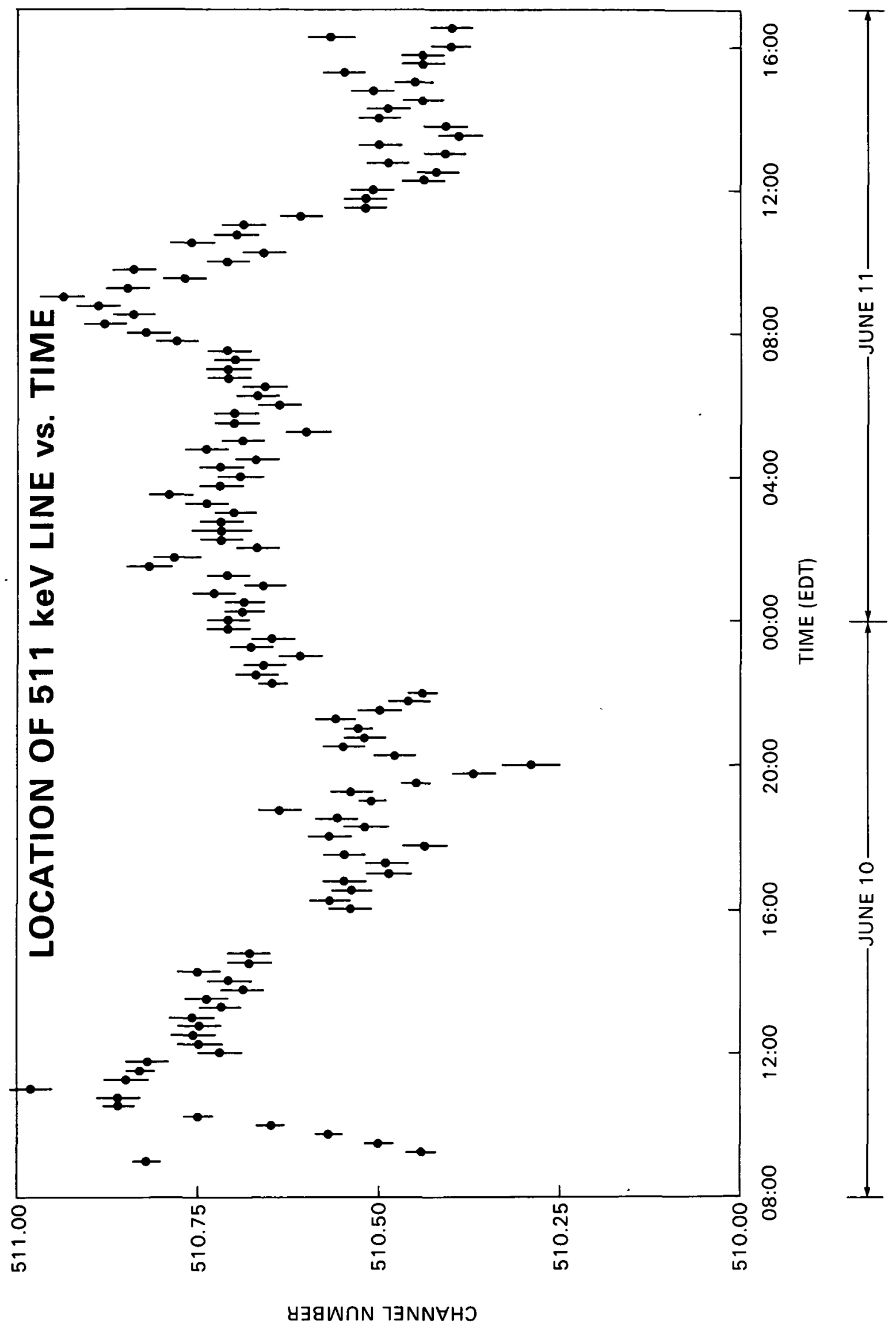


Figure 12

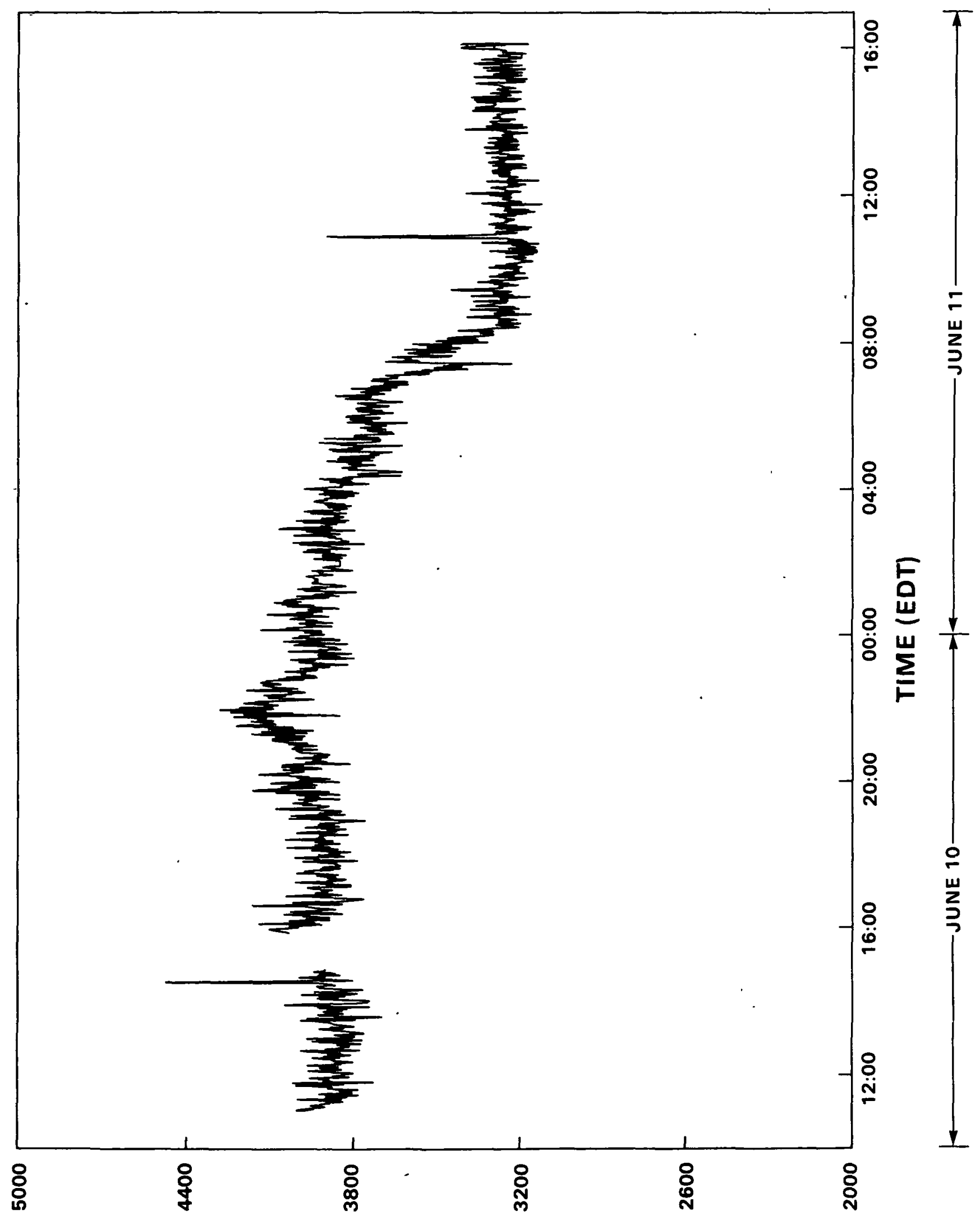

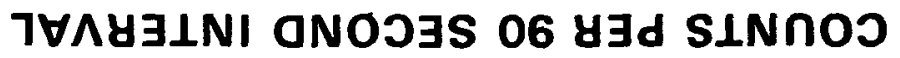


Figure 13

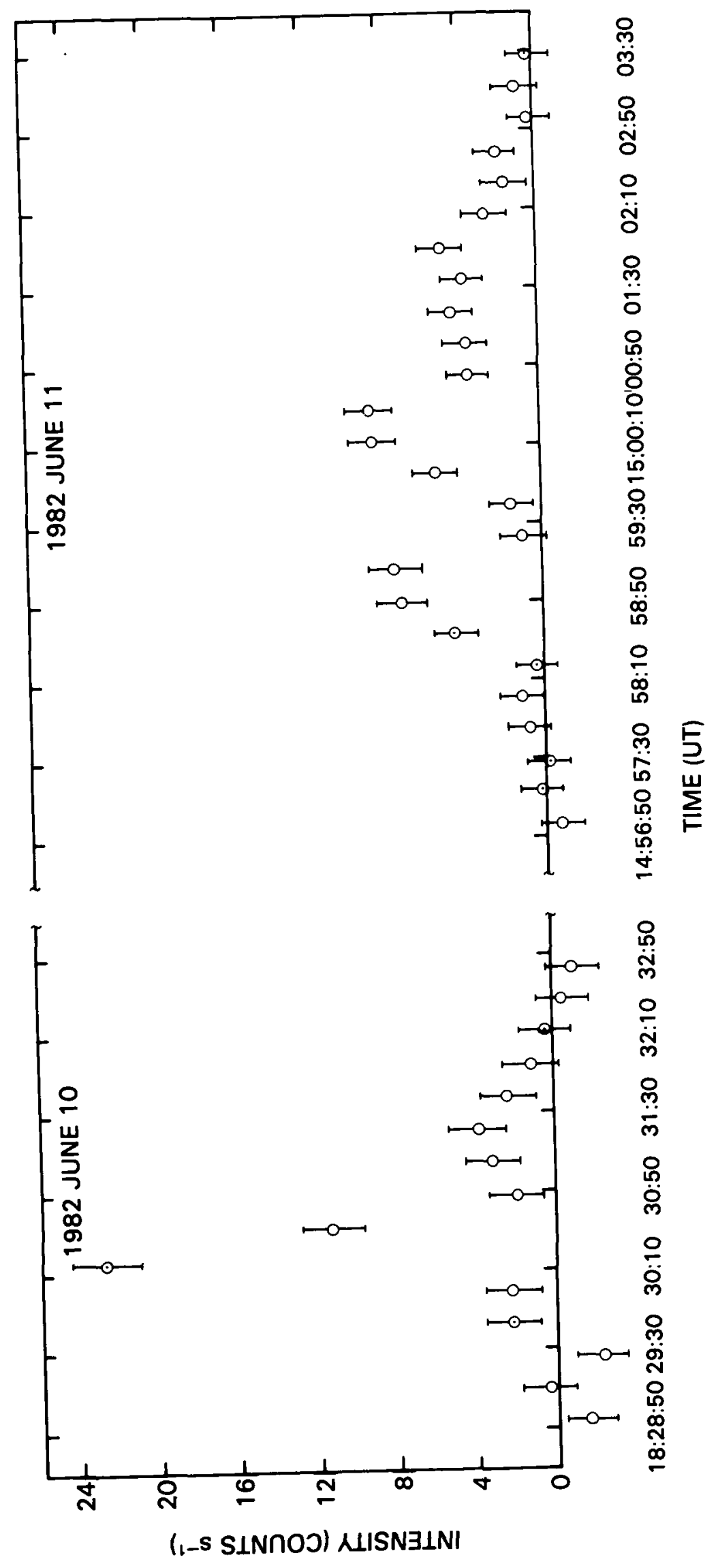


Figure 14

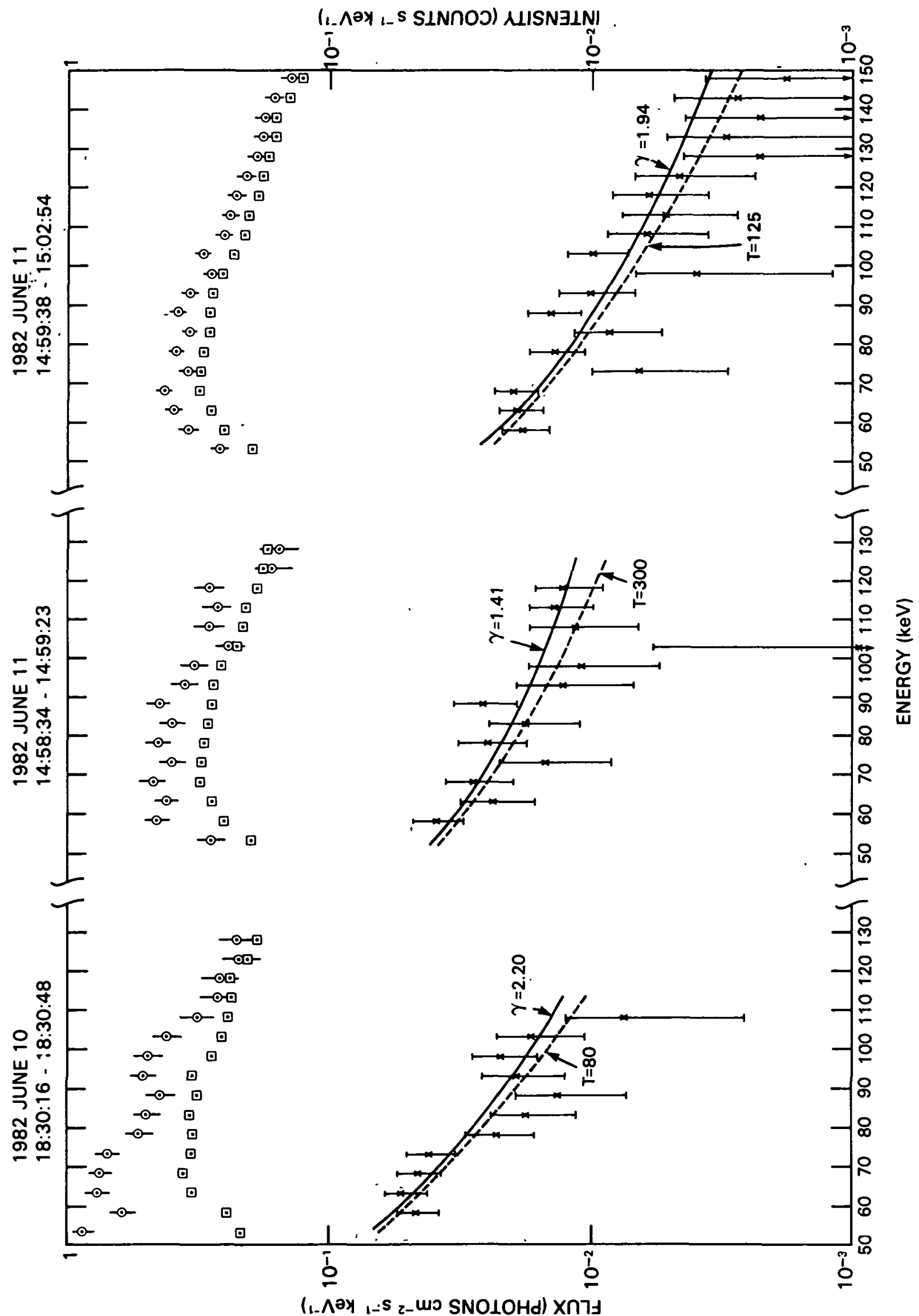


BIBLIOGRAPHIC DATA SHEET

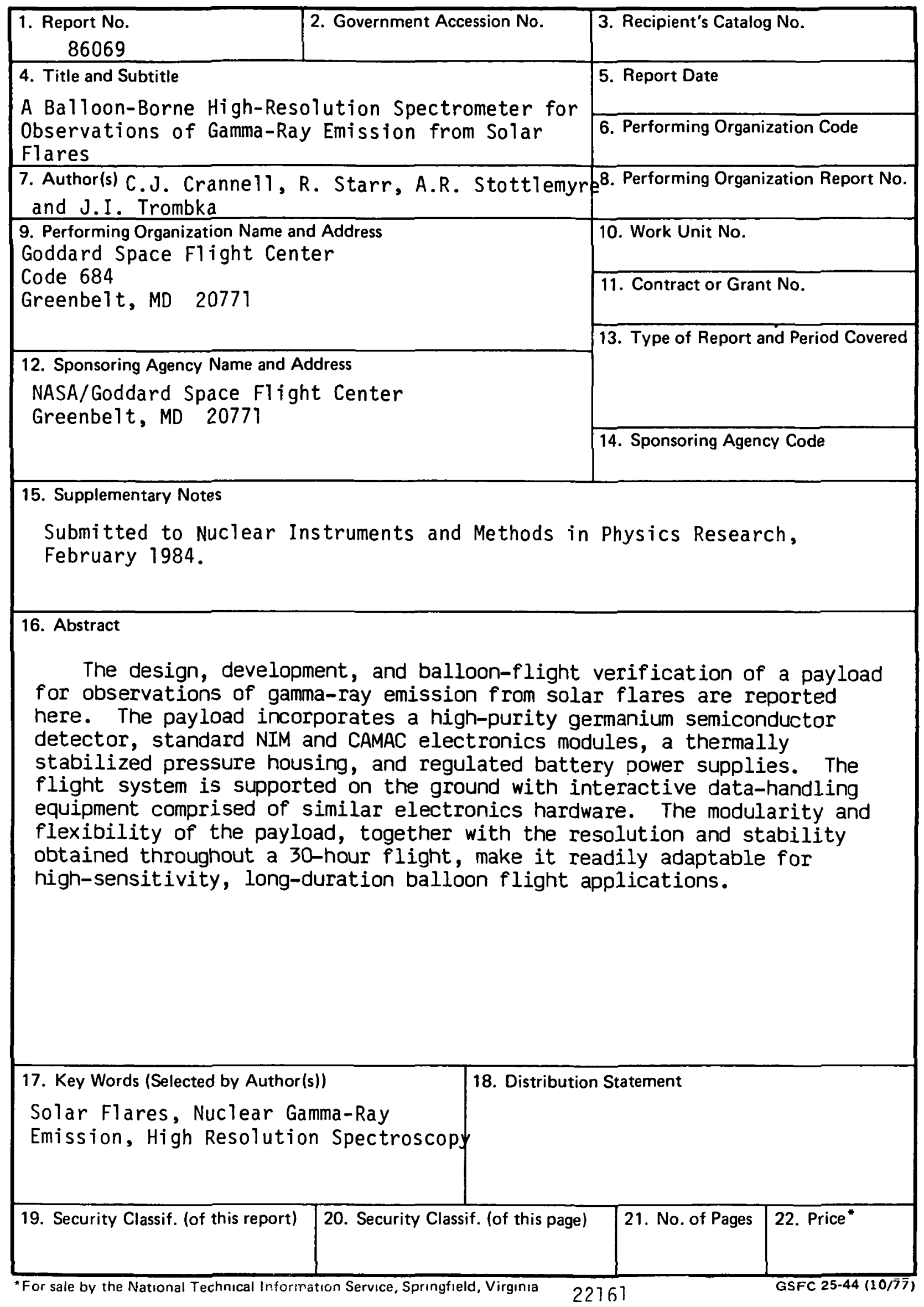

